\title{
The Australian Burden of Disease Study: impact and causes of illness and death in Aboriginal and Torres Strait Islander people, 2011
}

\author{
Fadwa Al-Yaman ${ }^{a, b}$
}

${ }^{a}$ Children's and Indigenous Group, Australian Institute of Health and Welfare, Canberra, ACT

b Corresponding author: fadwa.al-yaman@aihw.gov.au

\section{Article history}

Publication date: October 2017

Citation: Al-Yaman F. The Australian Burden of Disease Study: impact and causes of illness and death in Aboriginal and Torres Strait Islander people, 2011. Public Health Res Pract. 2017;27(4):e2741732. https://doi.org/10.17061/phrp2741732

\section{Key points}

- Burden of disease is higher among Indigenous Australians than among non-Indigenous Australians, with chronic disease contributing to $64 \%$ of the total burden of disease and $70 \%$ of the gap

- The burden of disease is largely due to the outcomes of modifiable risk factors

- There has been a small reduction in the overall burden since 2003

\section{Abstract}

This study estimates fatal and nonfatal disease burden among Indigenous Australians in 2011 and compares it with non-Indigenous Australians. The study found that there were 284 years lost per 1000 people because of premature death or living with ill health. Most of the disease burden was from chronic diseases (64\%), particularly mental and substance-use disorders, injuries, cardiovascular diseases, cancer and respiratory diseases. The burden of disease was higher among males (54\%) than females (46\%) and higher for fatal (53\%) than for nonfatal burden (47\%). The disease groups with the highest burden varied by age group, with mental and substanceuse disorders and injuries being the largest disease groups among those aged 5-44 years, and cardiovascular disease and cancer becoming more prominent among those aged 45 and older. Large disparities existed between Indigenous and non-Indigenous Australians, with the total burden being 2.3 times the non-Indigenous rates, fatal burden being 2.7 times and nonfatal burden being 2 times.

\section{Introduction}

There have been significant changes to the enumeration of the Indigenous population following the 1967 referendum. For example, the 1967 Australian Yearbook ${ }^{1}$ estimated the Aboriginal population at 79 253, of whom 39172 met the criteria for inclusion in national population counts and 40081 were 'full-blood'. In addition, there were 4972 full-blood Torres Strait Islanders and 245 half-European blood Torres Strait Islanders, giving a total Indigenous population of 84 470. Following the 1967 referendum, the Australian Census of Population and Housing moved to the 'origin' question (Are you of Aboriginal or Torres Strait Islander origin?), and the 1971 census was the first to use this question. The Aboriginal population was estimated at 115953 and 160915 in the 1971 and 1976 censuses respectively - an increase of $39 \%$ in 5 years. ${ }^{2}$ In the 2011 census, the Aboriginal and Torres Strait Islander 
population was estimated at 669900 , representing 3\% of the Australian population. ${ }^{3}$

The Royal Commission into Deaths in Custody national report ${ }^{4}$ recommendation 49 dealt with the paucity of statistical information about Aboriginal and Torres Strait Islander people, and stated that proposals for specific national surveys covering a range of health and economic characteristics of Indigenous Australians should be supported. Following this, the Australian Bureau of Statistics (ABS) was funded to undertake the National Aboriginal and Torres Strait Islander Social Survey, first conducted in 1994. Since then, the ABS has conducted an ongoing Indigenous-specific survey every 3 years, alternating health and social surveys. Significant progress has been made, and Indigenous identifiers have been progressively included in administrative data collections over time.

Key issues affecting existing administrative data collections are variation in the quality of Indigenous identification in these collections and changes in identification in the censuses over time. Also, for measures based on survey data, small sample sizes (which can lead to high variability) affect both the ability to disaggregate and the ability to monitor changes accurately.

\section{Indigenous burden of disease}

Burden of disease analysis is a way of measuring the impact of fatal and nonfatal diseases. It takes into account people's age at death and severity of disease, in addition to counting deaths and disease prevalence.

This paper presents the key findings from the most recent Australian Institute of Health and Welfare (AlHW) Burden of Disease Study of Aboriginal and Torres Strait Islander people. ${ }^{5}$ The study estimates the total disease burden (disability-adjusted life years or DALYs), fatal burden (years of life lost or YLL) and nonfatal burden (years lived with disability or YLD) of nearly 200 diseases and injuries for the Aboriginal and Torres Strait Islander population for 2011. The burden of disease is estimated by state and territory, region and socio-economic area of residence. The measures of disease burden in 2003 and 2011 are compared. The study uses and adapts the methods of global studies ${ }^{6}$ to produce estimates that are aligned with Australian health policy.

It is widely recognised that health and welfare outcomes are influenced by the broader social and economic conditions in which people live. ${ }^{7}$ Many of the issues experienced by Indigenous Australians are outcomes of a colonial history that separated Indigenous people from their land, resulting in a loss of lifestyle and culture and exposure to racism and discrimination, leading to social exclusion, marginalisation, oppression and poverty. A number of studies have found a link between trauma resulting from the intergenerational consequences of this history (or 'distal determinants') and poor health outcomes. ${ }^{8,9}$ The effects of distal determinants are difficult to measure because of their complex causal pathways, which in turn bring about poor outcomes for education, employment, housing, and ultimately health. The impact of the distal and socio-economic determinants on the burden of disease was not included in this study because of difficulty in obtaining good estimates of the risk associated with specific conditions.

Many datasets and epidemiological research studies are used in the burden of disease analyses. These include census data, Indigenous-specific and national survey data, administrative datasets (including perinatal, hospitalisation and mortality data), as well as a range of disease registers. Some of the data quality issues and limitations are outlined in the report, including the small sample size and the varying level of completeness of Indigenous identifiers in key datasets.

\section{Burden of disease estimates, 2011}

In 2011, the total disease burden among Indigenous Australians from living with ill health or dying prematurely was estimated at 190277 DALYs, representing a health loss of 284 years per 1000 Indigenous people. This loss was higher in males (54\%) than females (46\%) and increased with age. Chronic diseases were responsible for about two-thirds of the estimated burden - the leading causes of total health loss were mental and substanceuse disorders (19\%); injuries, including suicide (15\%); cardiovascular disease (12\%); cancer (9\%); and respiratory diseases (8\%).

A higher proportion of the disease burden was because of premature death (53\% or 100663 YLL; 150 YLL per 1000 people) than living with ill health (47\% or 89564 YLD; 134 YLD per 1000 people). The leading causes of the burden of living with ill health and of dying prematurely were different. Mental and substance-use disorders (39\%), musculoskeletal (14\%) and respiratory diseases $(12 \%)$ were the leading causes of living with ill health. Transport accidents and suicide (24\%), cardiovascular diseases (21\%) and cancer (17\%) were the leading causes of premature death.

\section{Relative burden is higher among Indigenous Australians}

The total burden of disease or health loss among Indigenous Australians was 2.3 times that of nonIndigenous Australians. The fatal burden and nonfatal burden were 2.7 and 2 times the non-Indigenous burden, respectively. Chronic diseases were responsible for $70 \%$ of the disease burden gap between Indigenous and nonIndigenous Australians: the highest contributors to these differences were cardiovascular diseases (19\%); mental and substance-use disorders (14\%); injuries, including suicide (14\%); respiratory diseases (10\%); cancer (9\%); and endocrine disorders, including diabetes (7\%). 


\section{Differences in disease burden by} age and sex

The total burden in young Indigenous children was relatively low, except where infant and congenital conditions were the predominant cause of the burden. Mental and substance-use disorders, and injuries (including suicide) were the main causes of burden for late childhood, adolescence and adulthood to age 44. Cardiovascular diseases and cancer start to emerge as major causes of the burden among Indigenous Australians from around age 45, and continue to be the main contributors to the disease burden in older Indigenous Australians. Respiratory diseases affected all age groups, accounting for between $4 \%$ and $13 \%$ of the total burden across age groups.

Figure 1. Total burden (number of DALYs), by disease group and age, Indigenous Australians, $2011^{5}$

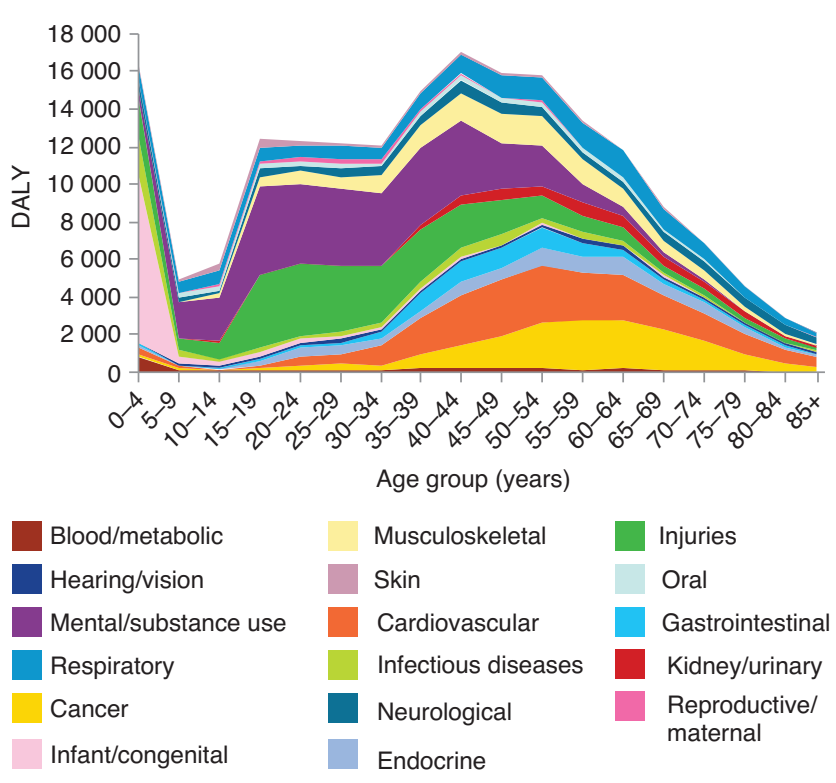

DALY = disability-adjusted life year

\section{Burden by state and territory}

The disease burden and its causes differed by state and territory, region and socio-economic area of residence. Indigenous Australians who lived in the Northern Territory (NT) and Western Australia (WA) had higher per person burdens of disease than those living in New South Wales (NSW) and Queensland. The age-standardised DALYs in NT and WA were 498.9 and 498.3 per 1000 people, respectively, and 408.7 and 419.2 per 1000 people for NSW and Queensland, respectively. These variations reflect not just differences in mortality and morbidity, but a complex interaction of other factors, such as demographic (including the age structure of the population and the proportion of the population that is Indigenous) and socio-economic variations. For example, the NT is quite different to other states and territories its population is younger, less likely to live in or near a capital city, and more likely to identify as Aboriginal or Torres Strait Islander.

Table 1. Age-standardised burden rates (per 1000 population) for Indigenous Australians, by state and territory, $2011^{5}$

\begin{tabular}{lccc}
\hline State or territory & Fatal burden & Nonfatal burden & Total burden \\
New South Wales & 227.8 & 180.9 & 408.7 \\
Queensland & 233.3 & 185.9 & 419.2 \\
Western Australia & 305.2 & 193.1 & 498.3 \\
Northern Territory & 328.5 & 170.3 & 498.9 \\
\hline
\end{tabular}

Note: Rates were age-standardised to the 2001 Australian standard population and are expressed per 1000 people.

The three leading causes of disease burden in NSW and Queensland were similar; the highest rates were for mental and substance-use disorders (21\% in both NSW and Queensland), followed by injuries, including suicide (12\% in NSW and 13\% in Queensland), and cardiovascular diseases (12\% in NSW and $11 \%$ in Queensland). In WA and the NT, the leading causes differed only slightly: injuries, including suicide, were the leading cause of the burden in both WA and the NT (19\% each), but mental and substance-use disorders were the second leading cause of burden for WA (17\%). Cardiovascular diseases were the second leading cause of disease burden in the NT (16\%). The third leading cause was cardiovascular disease for WA (14\%) and mental and substance-use disorders for the NT (13\%).

Figure 2. Leading causes of total burden of disease (percentage of DALYs), by state and territory, Indigenous Australians, $2011^{5}$

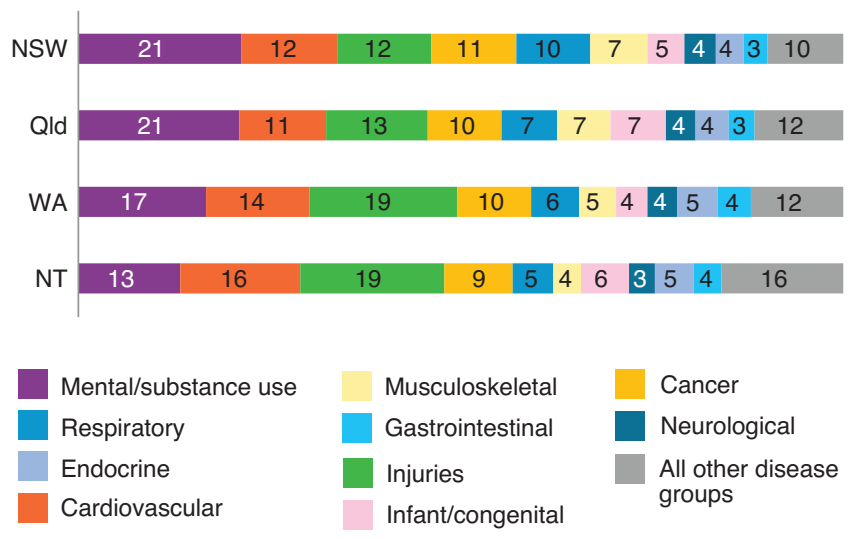

DALY = disability-adjusted life year 
The disease burden was substantially higher for people living in remote and very remote areas. Mental and substance-use disorders were the leading contributor to the overall disease burden in major cities, inner regional and outer regional areas. Injuries, including suicide, were the leading contributor to overall disease burden in remote and very remote areas. Rates of fatal burden were also highest in remote and very remote areas.

Those living in areas of high socio-economic disadvantage had 2.4 times the burden of those living in areas with the lowest socio-economic disadvantage. In each quintile of socio-economic status, the same disease groups were the leading contributors to the total disease burden - mental and substance-use disorders, injuries, cardiovascular diseases, cancer and respiratory diseases.

Between 2003 and 2011, a small 5\% reduction occurred in the disease burden (from 454 to 429 DALYs per 1000 Indigenous Australians, after adjusting for changes in the age of the population). This came mostly from reductions in cardiovascular diseases, infectious disease, endocrine disorders (which includes diabetes), gastrointestinal disorders, and infant and congenital conditions. This decrease in the total burden was mainly caused by a reduction in the fatal burden (11\%). The nonfatal burden increased by $4 \%$, because people are living longer with a disease rather than dying of it. Most of the decline in the fatal burden was because of a decline in mortality from cardiovascular diseases.

A substantial proportion (37\%) of the disease burden among Indigenous Australians is avoidable. The AlHW study examined the impact of 29 risk factors on the burden of disease, of which the leading contributors in 2011 were tobacco use (12\%), alcohol use (8\%), overweight and obesity (8\%), physical inactivity (6\%), high blood pressure (5\%) and high blood sugar (5\%), with a range of dietary factors combined contributing $10 \%$ (Table 2). Individual risk factors contribute to the burden for a number of diseases. For example, tobacco use causes around two-fifths of the burden from cancer, cardiovascular diseases and respiratory diseases respectively. The risk factors examined in this study were responsible for $50 \%$ of the gap in disease burden between Indigenous and non-Indigenous Australians, with tobacco use being the highest contributor to the gap (23\%).

\section{Conclusion}

This study found that chronic diseases were responsible for most of the disease burden among Indigenous Australians, and for most of the gap in the disease burden between Indigenous and non-Indigenous Australians. Mental and substance-use disorders caused most of the burden. The disease burden is largely preventable by addressing the risk factors associated with it. Although the study did not assess the contribution of social determinants to the disease burden because of a lack of relevant data and research, of those health risk factors examined, smoking was the highest contributor to the burden and made up almost one-quarter of the gap.

Table 2. Percentage of burden attributable to leading five risk factors for selected disease groups, Indigenous Australians, $2011^{5}$

\begin{tabular}{|c|c|c|c|c|c|c|c|}
\hline & Disease group & Tobacco use & High body mass & Alcohol use & $\begin{array}{l}\text { Physical } \\
\text { inactivity }\end{array}$ & $\begin{array}{l}\text { High blood } \\
\text { pressure }\end{array}$ & $\begin{array}{l}\text { Dietary risks } \\
\text { (joint effect) }^{\mathrm{a}}\end{array}$ \\
\hline $\begin{array}{l}\text { Proportion of } \\
\text { total burden }\end{array}$ & All diseases & 12.3 & 8.2 & 8.3 & 5.5 & 4.9 & 9.7 \\
\hline \multirow{7}{*}{$\begin{array}{l}\text { Proportion of } \\
\text { disease group } \\
\text { burden }\end{array}$} & Cancer & 39.0 & 5.3 & 2.8 & 4.8 & . & 9.6 \\
\hline & Cardiovascular & 39.4 & 33.8 & 3.3 & 28.8 & 35.4 & 50.1 \\
\hline & Mental & & & 22.2 & . & . & . \\
\hline & Injuries & & . & 18.5 & . & . & . \\
\hline & Respiratory & 41.7 & $\ldots$ & $\cdots$ & $\cdots$ & $\cdots$ & $\cdots$ \\
\hline & Endocrine & 8.2 & 62.3 & 0.7 & 35.6 & $\ldots$ & 60.7 \\
\hline & Kidney/urinary & . & 36.6 & . & . & 19.2 & $\ldots$ \\
\hline
\end{tabular}

Estimates for diet are based on an analysis of the joint effects of all dietary risk factors included in the study following methods used in recent global burden of disease studies. The estimates for different risk factors cannot simply be added to derive their total disability-adjusted life years, because of complex pathways and interactions between them.

Note: Blank cells '. .' indicate that the risk factor has no associated reference. 


\section{Acknowledgements}

This study was supported by an Indigenous Reference Group that included representatives from the departments of the Prime Minister and Cabinet, and Health, the National Aboriginal Community Controlled Health Organisation, and Indigenous members with expertise in data, epidemiology and health research. Regular updates about the study were also provided to the National Advisory Group on Aboriginal and Torres Strait Islander Health Information and Data.

\section{Competing interests}

None declared

\section{Author contributions}

FA-Y is the sole author of this article.

\section{References}

1. Commonwealth Bureau of Census and Statistics. Official yearbook of the Commonwealth of Australia: no. 53, 1967. Canberra: Commonwealth of Australia; 1967 [cited 2017 Sep 5]. Available from: http://www.ausstats.abs.gov. au/ausstats/free.nsf/0/E6D0D71B8E30099DCA257AF600 159B63/\$File/13010_1967_bk53.pdf

2. Australian Bureau of Statistics. Yearbook Australia: no. 65, 1981. Canberra: ABS; 1981 [cited 2017 Sep 5]. Available from: www.ausstats.abs.gov.au/ausstats/ free.nsf/0/68083A7E91641A24CA257AF700126CE2/\$Fi le/13010_1981_bk65.pdf

3. Australian Bureau of Statistics. Estimates of Aboriginal and Torres Strait Islander Australians, June 2011. Canberra: ABS; 2013 [cited 2017 Sep 5]. Available from: www.abs.gov.au/AUSSTATS/abs@.nsf/Lookup/3238.0.55. 001Main+Features1June\%202011?OpenDocument
4. Commissioner Elliott Johnston. Final report of the Royal Commission into Aboriginal Deaths in Custody. Canberra: Australian Government Publishing Service; 1991.

5. Australian Institute of Health and Welfare. Australian Burden of Disease Study: impact and causes of illness and death in Aboriginal and Torres Strait Islander people 2011. Canberra: AlHW; 2016 [cited 2017 Sep 5]. Available from: www.aihw.gov.au/getmedia/e31976fcadcc-4612-bd08-e54fd2f3303c/19667-bod7-atsi-2011. pdf.asp $x$ ?inline $=$ true

6. Institute of Health Metrics and Evaluation. The global burden of disease: generating evidence, guiding policy. Seattle: IHME; 2013 [cited 2017 Sep 5]. Available from: www.healthdata.org/sites/default/files/files/policy_ report/2013/GBD_GeneratingEvidence/IHME_GBD_ GeneratingEvidence_FullReport.pdf

7. Marmot MG and Wilkinson RG. Social determinants of health. 2nd ed. Oxford, UK: Oxford University Press; 2006.

8. Silburn S, Zubrick S, Lawrence D, Mitrou F, Blair E, Cox $A$, et al. The intergenerational effects of forced separation on the social and emotional wellbeing of Aboriginal children and young people. Canberra: Australian Institute of Family Studies; 2006 [cited 2017 Sep 5]. Available from: aifs.gov.au/sites/default/files/ss(2).pdf

9. Osborne K. Baun F, Brown L. What works? A review of actions addressing social and economic determinants of Indigenous health. Canberra: Australian Institute of Health and Welfare and Australian Institute of Family Studies; 2013 [cited 2017 Sep 5]. Available from: www.aihw.gov. au/getmedia/8eab67d7-1752-45e7-aa82-ffaf33ce7e13/ ctgc-ip07.pdf.aspx?inline=true

\section{Copyright: (c) (i) (-)}

(C) 2017 Al-Yaman. This article is licensed under the Creative Commons Attribution-NonCommercial-ShareAlike 4.0 International Licence, which allows others to redistribute, adapt and share this work non-commercially provided they attribute the work and any adapted version of it is distributed under the same Creative Commons licence terms. See: www.creativecommons.org/licenses/by-nc-sa/4.0/ 